\title{
La investigación escolar en educación básica para el desarrollo de competencias científicas en docentes ${ }^{1}$ School research in elemental education for the development of scientific competences in teachers
}

\author{
Diego Mauricio Luján-Villegas² (i), David Alberto Londoño-Vásquez ${ }^{3}$ (B)
}

2. Mg. Unidad Educativa San Marcos, Envigado, Colombia. Correo electrónico: dmlujanv@gmail.com

3. Ph.D. Institución Universitaria de Envigado, Envigado, Colombia. Correo electrónico:

dalondono@correo.iue.edu.co

Recibido en octubre 21 de 2019

Aceptado en abril 24 de 2020

Publicado en línea en agosto 25 de 2020

\section{Resumen}

Este artículo presenta la investigación escolar como una estrategia didáctica y metodológica en educación básica para el desarrollo de competencias científicas en docentes, puesto que requiere de la indagación, el fortalecimiento del pensamiento y la actitud crítica de ellos frente a sus prácticas en el aula. Aquí las competencias científicas se refieren a un proceso que permite entender la teoría desde una visión humanista con propósitos ciudadanos y participativos. Esto permite que se dé una formación que responda tanto a la teoría como a la práctica, de forma flexible, contextualizada y significativa, e impacta en sus participantes, no solo en lo disciplinario, sino en el desarrollo de competencias científicas que benefician su participación en la sociedad. Así, la investigación en la escuela se sale de lo curricular y se gesta desde las realidades y condiciones sociales y culturales de los implicados.

Palabras clave: competencias; competencias científicas; formación de docentes; investigación escolar.

\begin{abstract}
This paper presents school research as a didactic and methodological strategy in basic education for the development of scientific competences in teachers. Since it requires inquiry, the strengthening of teachers' thinking and critical attitude towards their practices in the classroom. Here, the scientific competences refer to a process that allows understanding the theory from a humanistic vision with citizen and participative purposes. That allows that the given training respond to both theory and practice, in a flexible, contextualized and meaningful way that affects its participants, not only in the disciplinary field, but also in the development of scientific competences that benefit their participation in society. In this way, research at school concentrates more on the realities and social and cultural conditions of those who are involved than in curriculum.
\end{abstract}

Key words: Competences; Scientific competences; Teacher training; School research.

\footnotetext{
${ }^{1}$ Este artículo se desprende de la investigación Desarrollo de Competencias científicas en maestros y maestras de la ciudad de Medellín que participaron en la feria CT+i ediciones 2012 a 2017. Un análisis desde la formación docente, realizada en el marco de la Maestría en Educación y Desarrollo Humano de la Universidad de Manizales-Cinde, aprobada en 2019.
} 


\section{Introducción}

a investigación en general se ha concebido como una Lactividad fundamentalmente prioritaria en la formación universitaria (Restrepo, 2004). Esto ha contribuido a que la investigación en las escuelas, especialmente en Latinoamérica, sea de interés de unos pocos (Bustos, 2011). En otras palabras, existe en el imaginario de las comunidades académicas que la investigación, como tal, solo puede realizarse a nivel universitario, lo que deja a un lado el potencial investigativo de la formación escolar primaria y secundaria (Arboleda, 2018).

Contemporáneamente, la formación de los docentes se considera como un factor que tiene una relación directa con los resultados de los estudiantes en su proceso de formación (Moreno, 2006), que ahora es medida por competencias (Martínez, 2004). Por tanto, se espera que los docentes de todos los niveles educativos cuenten con un proceso de formación continuo, que haga énfasis en la sistematización de sus prácticas y en la reflexión investigativa del entorno en el que se desarrolla el proceso de enseñanza y el de aprendizaje (Mejía y Manjarrés, 2011).

El punto de encuentro entre la investigación y la formación docente permite que se formulen preguntas frente al proceso de aprendizaje de los estudiantes de primaria y secundaria, y frente al desarrollo de competencias científicas que permitan estimular procesos investigativos (Londoño, Olave, Jaime y Losada, 2018), liderados por pares o docentes (Rodríguez y Díez, 2014), que además dinamicen la investigación docente o la investigación formativa (Valencia, Macías y Valencia, 2015). En otras palabras, existen competencias científicas que se desarrollan a partir de la investigación escolar que, aunque tienen una base común con la investigación, su finalidad las hace diferentes, pues la investigación per se busca producir conocimiento y la investigación escolar tiene como objetivo fortalecer las competencias científicas en la escuela, para la formación de ciudadanos críticos (Villalobos, Flórez y Londoño, 2017). Esto las hace pertinentes en el desarrollo de mejores condiciones para los estudiantes (Zabalza, 2007).

Por tanto, la investigación como estrategia pedagógica (Aglen, 2016) permite que las competencias científicas conocimiento, lenguaje y experiencia- (Roys-Romero, 2016) se desarrollen en tanto que, a partir de la conformación de comunidades de saber, el docente se configure como acompañante de procesos de investigación escolar, a partir de las preguntas que surgen tanto de la práctica del docente como de los aprendizajes del estudiante (Molina y Moreno, 2016).

Así, en este artículo se propone analizar las competencias científicas desde el contexto de la formación de docentes, instando a la posibilidad de su producción en la escuela como investigación escolar (Londoño y Luján, 2020), y a la formación de capacidades críticas (Martínez, Cabrera, Borjas, Torres y Judex-Orcasita, 2018) que permitan construir con los estudiantes diferentes apuestas desde las ciudadanías, incorporando el saber, el saber hacer, el ser y el aprender a vivir juntos (Quintanilla, 2005). Para ello se revisa aquí la investigación en clave de la escuela, las competencias en contexto, las competencias con enfoque científico y la formación de docentes en competencias científicas.

\section{La investigación escolar en educación básica}

La investigación escolar o investigación en la escuela es una estrategia didáctica y metodológica que propone una transformación de las prácticas pedagógicas a partir de la investigación como herramienta pedagógica. En ese orden de ideas, la investigación escolar busca el desarrollo de un pensamiento crítico en los estudiantes y el docente, quien acompaña este proceso que no solo desarrolla en los estudiantes habilidades de pensamiento, sino que también fortalece las competencias científicas y la formación de un mejor ciudadano.

La investigación escolar tiene una trayectoria amplia con algunos autores que son hitos importantes por sus aportes: Dewey (1933), Piaget (1935), Freinet (1962), Tonucci (1974), Allwright, Allwright y Bailey (1991), Greenhow, Robelia y Hughes (2009), entre otros. Su implementación compromete adecuaciones en el orden de lo epistemológico, lo didáctico y lo curricular. Siguiendo a Aguirre y Jaramillo (2008), hay que cambiar primero algunos paradigmas que han mistificado la investigación en el aula:

- Investigación por transmisión. El docente es el "sabelotodo" y el estudiante es quien recibe toda la información que este le puede compartir.

- La promesa mesiánica de la escuela. El aprendizaje se centra en la escuela, desconociendo el rol de los otros ciclos de socialización como la familia y los pares.

- Investigación como asignatura. La investigación se piensa en términos de currículo. Se "enseña" a partir de definición de conceptos. 
- La actividad científica tiende al descubrimiento de la verdad. Se considera la actividad científica como el medio para alcanzar "la verdad", desconociendo la característica de la construcción social de la ciencia.

- La semana de la ciencia. Mecanización y modelación de la investigación.

- Investigar un tema, más no un problema. Se desconoce el papel del problema frente a un tema de elección.

En virtud de lo anterior, se puede hablar de algunas maneras de propiciar la investigación en el aula. Una de ellas tiene que ver con la indagación como fuente de conocimiento que se centra en la pregunta como resultado de un proceso mediado por la curiosidad, que es aquella en la que se originan las reflexiones más profundas del ser humano. Unida a la pregunta está el aula como comunidad de indagación.

Otra forma de investigar desde el aula está relacionada con el fortalecimiento del pensamiento y la actitud crítica. Nada en la escuela debe ser considerado como dogma, y ninguna verdad acabada. Desde esta perspectiva, a través de la investigación se debe enseñar a pensar por sí mismo y a crear ideas propias, por encima de los modelos clásicos transmisionistas en los cuales los estudiantes y el maestro buscan certezas en los contenidos preestablecidos.

Si bien el estudiante es el protagonista del proceso de investigación escolar, para Cañal de León (1999):

Por lo que se refiere específicamente al profesor, su papel es el de un activo guía y compañero de investigación más experimentado, que ayuda impulsando las iniciativas de los alumnos y respetando un amplio margen para sus decisiones, pero también resaltando las contradicciones e incongruencias que percibe, que sus estudiantes pueden valorar como tales, remitiéndolos a la reflexión, al plan acordado y al compromiso con la coherencia. (p. 27).

En consecuencia, la investigación en la escuela se ha convertido en un reto para la comunidad educativa. Se percibe un desfase entre la enseñanza y la producción de conocimiento. Es decir, el docente no produce conocimiento como resultado de su práctica pedagógica, pues esta tarea le implica dedicar tiempo por fuera de su jornada laboral y un ejercicio riguroso de lectura y escritura que no siempre está a su alcance. Este aspecto marca una diferencia sustancial entre la investigación que se lleva a cabo en la formación universitaria y la que se pretende desarrollar en la básica y media; el docente en estos niveles atiende a unas lógicas diferentes al intelectual universitario o científico social y se debe dedicar a otra cosa, como el cuidado de los niños y la integración de las áreas.

Por último, para Restrepo (2004), la investigación que hace el docente sobre su práctica, la investigación que hace el docente sobre la práctica de los estudiantes y la investigación en la que el docente acompaña procesos investigativos de los estudiantes son las opciones a tener en cuenta en el aula. De las tres, la última es la de mayor recorrido en el medio, particularmente en Colombia, donde programas como Ondas y la Feria de la Ciencia, la Tecnología y la Innovación se han encargado de fortalecer el rol del docente acompañante (Colciencias-FES, 2009).

\section{Las competencias con enfoque científico}

En la misma línea, Quintanilla (2005) se refiere a las competencias científicas como una forma de comprender el contexto a partir de conceptos concebidos desde una visión humana de la ciencia, que va en pro de formar personas más críticas con alto grado de compromiso ciudadano. Por eso son necesarias tres cosas para construir competencias científicas: lenguaje, pensamiento y experiencia.

En este mismo sentido, se plantean tres dimensiones de las competencias científicas: conocimiento, habilidades y valores. Es decir, habilidades para conocer (saber) y saber hacer, y valores en el sentido de formar ciudadanos (Quintanilla, 2005). Por lo anterior, la perspectiva de trabajo desde el discurso de las competencias no puede desligarse del desarrollo de competencias ciudadanas: "el papel de la educación, tanto en la familia como en la institución educativa, es de gran importancia para desarrollar en la niñez y la juventud las competencias necesarias para desempeñarse de manera responsable en la vida pública y privada" (MEN, 2004, p. 153).

En Colombia, la formación por competencias en la educación científica surgió gracias al interés gubernamental centrado en las directrices de la globalización educativa, y a una apuesta decidida para su aplicación a nivel nacional. Por esta razón, al docente se le demandan competencias relacionadas con la contextualización de dichas directrices frente a la posibilidad de implementar en el aula actividades que permitan formar estudiantes desde el conocer, el ser, el hacer y el convivir, según lo propone la Organización de las Naciones Unidas para la Educación, la Ciencia y la Cultura 
(Unesco) (Delors, 1996). En otras palabras, es necesario, entender las competencias científicas como una serie de conocimientos, habilidades y capacidades que posibilitan comprender, analizar, proponer e interactuar proactivamente en la cotidianidad.

Al respecto, Carretero (2005) afirma que "las competencias científicas tienen que ver con la indagación, con la curiosidad, con pensar de una manera similar a la manera como piensan los científicos" (p. 92). Pero, posiblemente, una cuestión que se plantean todos los que trabajan en este ámbito es cómo conseguirlo, en otros términos, cómo conseguir una enseñanza que permita desarrollar esos cuatro aprendizajes que nos trae Delors (1996) en el informe para la Unesco: aprender a ser, aprender a hacer, aprender a conocer y aprender a vivir juntos.

Con base en lo anterior, definir las competencias investigativas del docente lleva consigo una comprensión profunda de la práctica pedagógica y de sus procesos, y la integración de los actores educativos. Por su lado, Borjas (2000) las explica como "las capacidades y actitudes del docente de someter a crítica lo que hace, cómo lo hace y cómo hacerlo mejor, con dominio de la lógica para resolver cualquier problema; es decir, en una reelaboración del saber" (p. 4). Esto exige una constante reflexión-acción de su práctica, una actitud crítica, para así discernir los modos más adecuados para mejorar la calidad de la educación y darle sentido a lo que realiza.

En este sentido, Muñoz, Quintero y Munevar (2001) plantean soluciones a los problemas detectados en el desarrollo científico, apuntando a la explicación, la interpretación y la crítica. Para estos autores existen dos dimensiones de las competencias científicas: una definida como dimensión pragmática que recoge la comprensión de competencia como habilidad que implica un conocimiento y un lenguaje; y la otra, como dimensión axiológica, en tanto se comprende que las competencias científicas no pueden estar aisladas de la formación ciudadana (competencias ciudadanas) y del desarrollo de valores que hacen de la ciencia un factor de desarrollo del sujeto para la transformación social.

La dimensión pragmática obedece a dos elementos esenciales cuando se hace referencia a las competencias: uno es el de habilidad, que se ubica en el saber hacer, y el de conocimiento, que se ubica desde el saber. Ambos están mediados por el lenguaje, en este caso, por el lenguaje científico. Eso permite acercarse a las competencias científicas desde una perspectiva que ubica al maestro como un profesional reflexivo que transforma su práctica y lo hace más competente.

La dimensión axiológica permite llevar las competencias al uso de las habilidades para la construcción de ciudadanía, esta entendida como un mínimo de humanidad compartida (MEN y FES, 1999). En ese sentido, lo que hay detrás es un sistema de valores desde el cual reconozco en el otro su humanidad y lo respeto a pesar de nuestras diferencias. Al pensar en competencias científicas está en medio no solo un sistema de valores para hacer juicios e identificar la veracidad de los argumentos, sino también la comprensión de un ser humano con valores que hace ciencia para generar nuevos valores y así transformar la sociedad.

A pesar de todo el influjo de las competencias en la educación, las críticas no se han hecho esperar por parte de estudiosos, académicos e investigadores del tema. Algunos las señalan como una "importación del mundo fabril" que se metió en el terreno de la educación, como si en educación también se comerciara con un producto de la misma forma que lo hacen quienes producen bienes y otros servicios. Otros consideran las competencias como una política expansionista del Banco Mundial y del Fondo Monetario Internacional con el fin de tener el control.

Vale la pena mencionar que este artículo difiere de tal postura, pues se entiende como una opción válida que integra los objetivos de formación en pro de evidenciar la relación entre lo aprendido, las habilidades para ejecutarlo, el desarrollo humano y la posibilidad de convivir en sociedad.

\section{La formación de docentes en competencias científicas}

Martínez (2004) centra el interés en la formación docente desde la perspectiva del discurso de las competencias. Esta formación, desde donde quiera que se mire, agrupa el conocimiento de la disciplina, la fundamentación pedagógica disciplinaria, la estructuración didáctica, las habilidades comunicativas tanto con los estudiantes y familiares como con sus colegas y directivos, y la capacidad de contextualización social y cultural en el aula. Esto le permitirá al docente trabajar en equipo con los colegas a partir de un lenguaje común y, desde luego, para todo esto necesita un conocimiento práctico que es algo más o menos como saber hacer las cosas bien en lo cotidiano. Algunos autores, afirma Martínez (2004), reemplazan "conocimiento" por competencia, pero en última instancia la competencia es el 
uso práctico de un conocimiento, por tanto, no son términos disyuntivos, pues ponen en juego la formación del maestro para la autonomía o para la sumisión a partir de deseos, saberes y lenguajes.

Con base en lo anterior, surge desde la academia y la vida cotidiana una epistemología en clave constructivista que se basa, según el mismo autor, en los siguientes criterios:

- La práctica es un escenario complejo, incierto y cambiante que no solo se basa en la teoría, pues allíse producen interacciones entre los sujetos que es preciso observar, cuestionar y reformular. La interacción entre teoría y práctica permite expandir el conocimiento y transformar la realidad y, como consecuencia de esta interacción, se transforma el sujeto.

- Los seres humanos construyen significados y representaciones que se construyen a partir de los modelos mentales, las creencias socialmente aceptadas, las identidades y la emoción.

- Aprender supone reconstruir, reestructurar, redescubrir de forma sistemática y consciente el tejido de dichas representaciones en cada sujeto.

- El conocimiento permite indagar por nuevas respuestas a las preguntas, identificar nuevas preguntas y formas de responderlas, intentar resolver problemas que recién surgen 0 tienen otras causalidades, en pro de brindar mayor bienestar constantemente a la sociedad.

En otras palabras, la formación de docentes supone el desarrollo de los procesos de interacción entre teoría y práctica. La formación de este pensamiento práctico del docente, sus cualidades humanas fundamentales, requieren entender sus teorías personales.

En este sentido, la formación de los maestros no solo implica un proceso profesionalizante circunscrito únicamente a la formación intelectual, pues la experiencia cotidiana también juega un papel fundamental donde el aula de clase se convierte para el maestro en un escenario propicio para su formación. Por eso es importante darle valor a su experiencia, a sus relatos, a sus historias y vivencias que han marcado la práctica pedagógica.

De acuerdo con Pérez (2010), son tres los grupos de competencias en los que se basan la mayoría de los programas de formación de maestros:
- Planear, proponer y valorar las prácticas pedagógicas que fomentan el desarrollo humano.

- Generar escenarios de socialización en pro de la construcción de la democracia, el reconocimiento de las diferencias y el respeto de ellas.

- Propender por el desarrollo profesional, la participación en redes y grupos de pares.

En lo que se refiere al primer elemento, es necesario ir más allá de la simple historiografía para identificar los imaginarios culturales existentes en torno a la figura del maestro que vinculan lo teórico y lo práctico.

El segundo elemento tiene relación con la pedagogía como saber fundante, entendido como la capacidad que el docente tiene de enseñar, y como teoría de la formación. En esta línea, en los aparatos disciplinarios que estructuran la formación hay dos ámbitos: uno conformado por aquellas disciplinas que contribuyen a dar sentido y a estructurar la identidad profesional (pedagogía, didáctica, historia de la enseñanza, psicología evolutiva y epistemología) y otro que aporta a la comprensión del contexto en el que se ejerce el oficio de maestro (historia de la educación, antropología, filosofía, sociología, entre otros).

Es necesaria la capacidad de superar lo mítico a través de la crítica que se fundamenta en la reflexión seria y sistemática que hacen los maestros a partir de sus prácticas cotidianas. Para ello es necesario recuperar elementos sustantivos de la tradición, actualizándolos, indagando en los escritos de los viejos maestros aquellas ideas que pueden cobrar significado en la escuela de hoy.

En definitiva, la formación de docentes en competencias científicas compromete las dimensiones epistemológica y pedagógica que integran lo teórico, el docente como intelectual y lo práctico, el docente como constructor de saberes a partir de su experiencia. En este sentido, también se involucran otras disciplinas como la psicología, la sociología, la antropología, la filosofía, entre otras.

\section{Conclusiones}

La investigación escolar cuenta con una trayectoria importante. No obstante, parece ser desvirtuada en el contexto de la praxis docente en primaria y secundaria, y se relega a escenarios de formación universitaria donde es realizada por especialistas temáticos. Lo contrario invitaría a la desmitificación de algunos paradigmas de la investigación 
escolar, donde se empodere al estudiante como agente de generación de conocimiento, se saque al estudiante de la formación primordialmente in situ, se dinamice la formación en investigación a través de la práctica, y se permita que el docente reflexione y ponga en duda la información revisada, y aprenda a problematizar los elementos que giran en su entorno.

Para ello, una forma de propiciarlo es llevando a cabo un proceso de formación concentrado en la pregunta, la indagación y el pensamiento crítico. En otras palabras, se propicia permitiéndole al estudiante preguntarse por las posibles causas sin una previa inducción, acompañándole en los procesos de indagación que le permitan recolectar la información y acceder a los posibles conceptos o temáticas, para que a partir de la reflexión teórica pueda poner en duda lo enseñado y crear puntos de encuentro entre la información y los conceptos.

Esto es factible a través de la formación por competencias, concepto que emigró del mundo laboral a la educación, gracias a las posibilidades de evidencia del desarrollo de capacidades en el ser, el saber y el saber hacer desde una perspectiva constructivista; a la problematización como eje de formación y a la argumentación como medio de ilación teórica, conceptual y epistemológica, que pone en crisis la transmisión de información y la memorización de conceptos: habilidades extremadamente sobrevaloradas en la educación tradicional.

Estas competencias pueden ser científicas, las cuales no solo reconocen la importancia de las prácticas pedagógicas apuntadas hacia el desarrollo de un pensamiento crítico y el raciocinio, sino que también propenden por la construcción de habilidades para la vida, el reconocimiento del otro y del entorno, y el afianzamiento de prácticas ciudadanas correspondientes a las necesidades del presente siglo. Por tanto, las competencias científicas pueden entenderse como un cúmulo de saberes, capacidades y disposiciones que permiten la acción y la participación del estudiante de manera significativa en su entorno.

Finalmente, la formación de docentes en competencias científicas requiere de aspectos como el dominio temático por parte del docente, con capacidad pedagógica para compartir dicho tema bajo una creatividad didáctica y habilidades comunicativas que propendan por la solución de problemas y el trabajo en equipo. Estos aspectos no solo le permiten al docente generar espacios propicios para el desarrollo de competencias científicas de sus estudiantes, sino también la continua reconstrucción conceptual, la resemantización de las experiencias y la contextualización del conocimiento investigativo.

\section{Declaración de conflictos de intereses}

Los autores manifiestan que durante la ejecución del trabajo - la redacción del artículo no han incidido intereses personales o ajenos a su voluntad, incluyendo malas conductas y valores distintos a los que usual y éticamente tiene la investigación.

\section{Referencias bibliográficas}

Aguirre, J. C. y Jaramillo, L. G. (2008). Consideraciones acerca de la investigación en el aula: más allá de estar a la moda. Educación y Educadores, 11(1), 43-54. Recuperado de http://www.scielo.org.co/scielo.php?script=sci arttext\&pi $\mathrm{d}=$ S0123-12942008000100004\&lng=en\&tIng=es

Aglen, B. (2016). Pedagogical strategies to teach bachelor students evidence-based practice: A systematic review. Nurse education today, 36, 255-263. Recuperado de http://www.sciencedirect.com/science/article/pii/S02606 $\underline{91715003494}$

Arboleda, J. C. (2018). Tomo XIV: formación, investigación y ambientes de aprendizaje. Boletín Redipe, 7(6), 135-142. Recuperado de http://dialnet.unirioja.es/servlet/ articulo? codigo $=6523269$

Allwright, D., Allwright, R. y Bailey, K. M. (1991). Focus on the language classroom: An introduction to classroom research for language teachers. Cambridge, England: Cambridge University Press.

Borjas, B. (2000). El docente como investigador. Revista Movimiento Pedagógico, 2, 34-47. Recuperado de https://www.movimientopedagogico.com.ve/revista.php

Bustos, A. (2011). Investigación y escuela rural: ¿irreconciliables? Profesorado. Revista de currículum y formación de profesorado, 15(2), 155-170. Recuperado de http://www.redalyc.org/pdf/567/56719129011.pdf

Cañal de León, P. (1999). Investigación escolar y estrategias de enseñanza por investigación. Revista Investigación en la Escuela, 38, 15-36. Recuperado de http://idus.us.es/xmlui/ handle/11441/60091

Carretero, M. (2005). Constructivismo y Educación. Buenos Aires, Argentina: Editorial Paidós. 
Colciencias-FES. (2009). Reconstrucción colectiva del Programa Ondas (2006-2008). La Investigación como Estrategia Pedagógica. Bogotá: Colciencias.

Dewey, J. (1933). Cómo pensamos. Barcelona, España: Paidós.

Delors, J. (1996). La educación encierra un tesoro. Informe a la Unesco de la comisión Internacional sobre la educación para el siglo XXI presidida por Delors. Madrid, España: Santillana.

Freinet, C. (1962). La enseñanza de las ciencias. Barcelona, España: Laia.

Greenhow, C., Robelia, B. y Hughes, J. E. (2009). Learning, teaching, and scholarship in a digital age: Web 2.0 and classroom research: What path should we take now? Educational Researcher, 38(4), 246-259. Recuperado de http://journals.sagepub.com/doi/abs/10.3102/0013189X0 9336671?journalCode=edra

Londoño, D., Olave, J., Jaime, J. y Losada, N. (2018). Lógicas, enfoques y epistemologías sobre educación y pedagogía. En H. Ospina y otros. Educación y Pedagogía. Trayectos recorridos (pp. 96-155). Manizales, Colombia: Universidad de Manizales.

Londoño, D. y Luján, D. (2020). Competencias científicas en maestros de la ciudad de Medellín. Cultura, Educación y Sociedad, 11(1), 39-54. Recuperado de https://revistascientificas.cuc.edu.co/culturaeducacionyso ciedad/article/download/2719/2658?inline=1

Martínez, J. (2004). La formación del profesorado y el discurso de las competencias. Revista Interuniversitaria de Formación del Profesorado, 18(3), 127-143. Recuperado de http://ucsj.redalyc.org/articulo.oa?id=27418308

Martínez, A., Cabrera, H., Borjas, M., Torres, E. y JudexOrcasita, J. (2018). Evaluando la disposición y la motivación del pensamiento crítico con la mediación de las TIC. Praxis, 14(2), 187-203. Recuperado de http://revistas.unimagdalena.edu.co/index.php/praxis/art icle/view/2762/2072

Mejía, M. y Manjarrés, M. (2011). La investigación como estrategia pedagógica una apuesta por construir pedagogías críticas en el siglo XXI. Praxis \& Saber, 2(4), 127177. Doi: https://doi.org/10.19053/22160159.1127

Moreno, J. M. (2006). Profesorado de Secundaria y Calidad de la Educación: Un marco de opciones políticas para la formación y el desarrollo profesional docente. Profesorado. Revista de Currículum y Formación de Profesorado, 10(1), 1-20. Recuperado de http://www.redalyc.org/pdf/567/ 56710104.pdf
Ministerio de Educación Nacional (2004). Estándares Básicos de Competencias Ciudadanas. Bogotá, Colombia: ICFES.

Ministerio de Educación Nacional e Instituto Colombiano para el Fomento de la Educación Nacional (1999). Examen de Estado para el ingreso a la Educación Superior. Cambios para el Siglo XXI. Propuesta General. Bogotá, Colombia: ICFES.

Molina, Y. A. y Moreno, C. P. (2016). La esencia de la formación de formadores en el contexto de la educación a distancia. Praxis, 12, 111-117. Recuperado de http://revistas.unimagdalena.edu.co/index.php/praxis/art icle/view/1852/1330

Muñoz, J., Quintero, J. y Munevar, R. (2001). Competencias Investigativas para profesionales que forman y enseñan. Bogotá, Colombia: Editorial Magisterio.

Pérez, Á. I. (2010). Aprender a educar: nuevos desafíos para la formación de docentes. Revista interuniversitaria de formación del profesorado, (68), 37-60. Recuperado de http://dialnet.unirioja.es/servlet/articulo?codigo $=327604$ $\underline{4}$

Piaget, J. (1935). El juicio moral en el niño. Madrid, España: Francisco Beltrán.

Quintanilla, M. (Ed.), (2005). Enseñar ciencias en el nuevo milenio. Retos y propuestas. Santiago, Chile: Ediciones Universidad Católica de Chile.

Restrepo, B. (2004). La investigación-acción educativa y la construcción de saber pedagógico. Educación y Educadores, 7, 45-55. Recuperado de http://www.autores. redalyc.org/articulo.oa?id=83400706

Rodríguez, C. y Díez, J. (2014). Conocimiento y competencias básicas en la formación de maestros y maestras. Profesorado, Revista de Currículum y Formación del Profesorado, 18(1), 383-396. Recuperado de http://recyt.fecyt.es/index.php/profesorado/article/view/ $\underline{41092}$

Roys-Romero, N. R. (2016). Competencias docentes: Desde una perspectiva etnoeducativa y tecnológica. Praxis, 12, 7889. Recuperado de http://revistas.unimagdalena.edu.co/ index.php/praxis/article/view/1849

Tonucci, F. (1974). La escuela como investigación. Barcelona, España: Avance.

Valencia, J., Macías, J. y Valencia, A. (2015). Formative research in higher education: some reflections. ProcediaSocial and Behavioral Sciences, 176, 940-945. Recuperado de http://www.journals.elsevier.com/procedia-social-and$\underline{\text { behavioral-sciences }}$ 
Villalobos, J., Flórez, G. y Londoño, D. (2017). Relación maestro-alcance del logro: Una mirada a los procesos de enseñanza en la escuela. Entramado, 13(1), 186-196. Recuperado de http://www.scielo.org.co/scielo.php?pid=S 1900-38032017000100186\&script=sci abstract\&tlng=en
Zabalza, M. (2007). Competencias docentes del profesorado universitario. Calidad y desarrollo profesional. Madrid, España: Narcea.

Para citar este artículo: Luján-Villegas, D. M. y Londoño-Vásquez, D. A. (2020). La investigación escolar en educación básica para el desarrollo de competencias científicas en docentes. Praxis, 16(2), 227-234. Doi: https://doi.org/10.21676/23897856.3276 